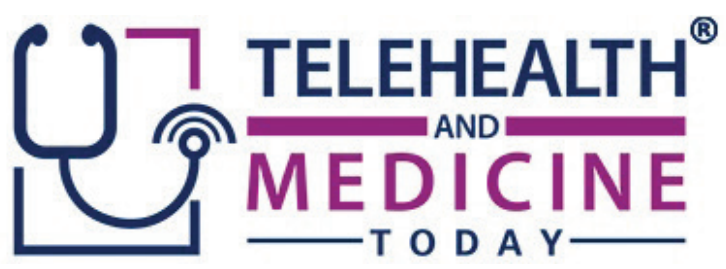

\title{
ConV2X 2019 Innovation Ignition Pitch Competition: An Interview with Award Recipient, Rachel Phillips, Founder, Med-Rok Systems Inc.
}

Tory Cenaj ${ }^{1}$, Rachel Phillips ${ }^{2}$

Affiliations: ${ }^{1}$ Partners in Digital Health, Stamford, CT; ${ }^{2}$ Med-Rok Systems Inc., Santa Monica, CA

Corresponding Author: Tory Cenaj, teecellc@gmail.com

Keywords: AdmitScan, Blockchain, ConV2X, ConVerge2Xcelerate, Med-Rok Systems, Telehealth, Transpedite

Section: Opinions, Perspectives, and Commentary on a current trend or issue impacting the sector

The ConV2X Pitch Competition was held in Boston on October 15, 2019 during the ConVerge2Xcelerate (ConV2X) Third Annual Meeting. Six finalists presented their products to judges who were associated with Flare Capital, Pillar VC, Curisium, Harvard University, and UC San Diego School of Medicine.

Their challenge was to "Prove Market Value"the theme of ConV2X 2019-and demonstrate comprehensive market solutions with compelling value propositions for stake holders, including providers, payers, pharma, employers, and consumers.

This interview features Rachel Phillips, Founder, Med-Rok Systems Inc. and Tory Cenaj, Publisher, Telehealth and Medicine Today.

\section{Ms. Cenaj: Why did you enter the ConVerge2Xcelerate (ConV2X) 2019 Competition?}

Ms. Phillips: Our products are Transpedite and AdmitScan. And we were seeking funding for a 4-month pilot with a group of 5 hospitals, 15 discharge facilities, 2 shelters, and 1 telehealth company. We also wanted exposure for our solutions, make important connections, and learn more about blockchain and telehealth technologies.

\section{Ms. Cenaj: Why are competitions important} to startups?

Ms. Phillips: It's all about marketing, establishing connections, and gaining access to investors.

Ms. Cenaj: Tell us a bit about your product. What market gap does it fill?

Ms. Phillips: We examined outside industry's affordable and successful use of web technology 
that utilizes a unified platform to connect disparate entities to each other and to their consumers while automating the processes between them. Examples include Expedia, Trivago, Uber, and Amazon. We then applied a combination of these functions to solving health care's disconnected and disorganized processes.

Transpedite automates placement of hospital patients to skilled nursing facilities and shelters along with the process of setting up home health, hospice, durable medical equipment, and ambulance transports, cutting the current process from 2 to 14 days down to minutes using one-fourth of the workforce.

For insurers, Transpedite offers automated insurer authorizations or letters of agreement, which are necessary before a patient can be placed. By comparison, the current process requires hundreds of insurer case managers to review clinical information, with delays of up to 2 weeks before securing authorization.

The system includes a patient/family module, which provides video-education on levels of facility care, as well as searching and viewing virtual visit videos of available facilities while assigning preference ratings. During the process, the patient and family are updated on the transfer progress in real time. This capability also decreases discharge delays and workforce, while improving patient/family satisfaction with the hospital care. In addition, telehealth appointments can be scheduled for high-risk readmission cases to decrease skilled nursing readmission rates.

Our second web-based product is AdmitScan, which effectively eliminates surprise billing.

\section{Ms. Cenaj: What is the economic impact to the healthcare system and health consumer? And, are outcomes documented using outcomes data?}

Ms. Phillips: Once the physician assesses a patient as no longer needing hospital level of care, the insurer stops reimbursements. The national average discharge delay of hospital patients to skilled nursing facilities is 3.5 days and a shocking 8 days to find shelter placement for homeless patients. This means that not only is the hospital losing income due to costs associated with continuing care for a non-paying patient, but the institution is also foregoing $\$ 3,000$ (for med-surg level care) to $\$ 12,000$ (for intensive care) per day from a paying patient who could occupy that bed. Discharge delays can lead to \$14 to \$22 million annual losses for each hospital.

Currently, hospital case management department workforce budgets are costly (up to $\$ 3$ to $\$ 4$ million for hospitals with 400 beds or more) as many workers are needed to make hundreds of manual phone calls and repeated faxing/emailing to facilities in order to secure a bed and coordinate the transfer with patients and their families. The use of Transpedite will cut workforce requirements in half.

\section{Ms. Cenaj: What is your business model?}

Ms. Phillips: Our SAAS model for Transpedite is a $\$ 120,000$ annual subscription for hospitals (the yearly cost of a single case manager). For skilled nursing facilities, it is $\$ 30,000$ annually (half the cost of a single marketing associate to secure patients) to keep their beds filled with "profitable" cases. Insurer's cost is $\$ 200,000$ annually (the cost of two insurer case managers) to provide efficient automated authorizations for hospital transfers. Shelters use Transpedite for free. 
Our SAAS model for AdmitScan is $\$ 1.00$ per month per member and their first dependent and a $\$ 0.25$ per month for additional dependents. This means that a family of four can be protected from surprise billing and remain within their cost-effective networks for $\$ 30$ per year.

\section{Ms. Cenaj: How do we increase the use of digital tech with heath consumers?}

Ms. Phillips: The healthcare industry has focused primarily on consumer tech that offers vital sign monitoring, exercise tracking, and patient portals to view lab results or schedule appointments. These "novelty tech toys" are not proven to improve costs or health outcomes.

We focus on integrating the healthcare consumer into their actual healthcare process, with the ability to communicate, share, and coordinate their healthcare activities with their physicians, hospitals, and insurers in real time. This offers the consumer real cost and quality benefits.

For example, our AdmitScan is an insurer-interfaced member QR code that when scanned in any emergency department (ER), admitting department, or physician office notifies the payer, the primary care physician, and the medical management entity of all emergency department visits or hospital admissions. This allows the primary care provider to intervene in a timelier manner with telehealth or appointments to address/adjust the treatment plan. The scan also eliminates surprise billing by instantly notifying the consumer, hospital, and physician if the plan is out-of-network, with an electronic process to transfer the patient back to network care-effectively eliminating surprise billing, which contributes to consumer bankruptcy.

Ms. Cenaj: What educational tools do we need? Ms. Phillips: We need tools that are interactive and, again, integrate the consumer into the process with multiple providers as care is happening. This might include wireless home biometric monitoring of glucose, heart rate/ rhythms, and blood pressure that connect abnormal values in real time to primary care physicians who can quickly contact the patient by telehealth or phone to adjust medications or treatment plans.

\section{Ms. Cenaj: What are your predictions for telehealth over the next couple of years?}

Ms. Phillips: Telehealth use will increase greatly. It is already used in hospitals that lack certain specialists to evaluate psych or neuro cases; and now telehealth in skilled nursing facilities is helping decrease high readmission rates.

I would like to say that the success of telehealth will lie in the quality of the physicians who provide such evaluations, as I have been in some hospitals where the telehealth consult was so generic and lacking in treatment plan or follow-up that we found them useless.

The other issue with telehealth is that data aren't being shared between telehealth companies. For example, if a patient has a telehealth visit for a urinary tract infection and is prescribed an antibiotic, they could essentially have multiple telehealth consults with other companies for the same issue without the provider knowing they had obtained an antibiotic through a previous provider. This is worrisome when it comes to the rise of resistant bacteria.

\section{Ms. Cenaj: Do you think patients should own their data?}

Ms. Phillips: Patients already own their data. If they want to obtain a copy of their medical records, patients should contact their doctor's office, clinic, or hospital where they were treated. 
The real issue is that obtaining that information is cumbersome and the information is spread out among multiple providers. We need the ability to consolidate and centralize records for consumers, as well as providers.

\section{Ms. Cenaj: How can the government help startups? And do you want their help?} Ms. Phillips: With Transpedite, we are trying to contact Medicare and Medicaid to improve their member costs through efficient discharges to skilled nursing and shelters with automated authorizations.

We have contacted US senators on the committee charged with addressing surprise medical billing with regard to AdmitScan, which effectively eliminates the surprise billing issue. The committee is proposing a solution that completely exempts out-of-network consumers from any out-of-network costs, which will shift these costs and losses to hospitals and physicians. This will cause hospitals/physicians to raise their prices, which will cause insurers to raise premiums, ultimately harming all consumers. AdmitScan preserves our current system of cost-effective networks without negative repercussions. We would like to present our AdmitScan solution to the Senate.

\section{Ms. Cenaj: We hear about agnostic platforms and their ability to provide users the broadest access to quality care. How is that effort progressing?}

Ms. Phillips: Our web-based products are agnostic platforms in the sense that they can share data with current electronic health records (EHR) without expensive integration or maintenance costs.

Our future goal is to shift to a secure web-based EHR that goes beyond record keeping and data sharing in order to analyze data in real time and contribute to the team's care quality. We would like to construct a mobile, voice-activated EHR documentation system that removes the healthcare team from their computer screens and keyboards, returning them to hands-on bedside care.

\section{Ms. Cenaj: What role do you think peer-review journals fill in the marketplace? Is there still a place for them? \\ Ms. Phillips: Peer-review journals are extremely important for sharing innovation and uniting healthcare entrepreneurs globally.}

\section{Ms. Cenaj: Moving forward, what technologies will be most useful to health care in reducing costs?}

Ms. Phillips: Our healthcare industry has struggled for years to coordinate services and products using expensive, complex integration, and unactualized interoperability of health records.

We must start using "outside industry" technologies that offer web-based affordable and successful systems. This allows for the real-time integration of consumers into their healthcare process. Our web-based products exceed industry security standards and are protected by HIPAA managed servers, HTTPS encryption, VPN (virtual private network), and onsite RSA authentication.

Copyright Ownership: This is an open access article distributed in accordance with the Creative Commons Attribution Non Commercial (CC BY-NC 4.0) license, which permits others to distribute, adapt, enhance this work non-commercially, and license their derivative works on different terms, provided the original work is properly cited and the use is non-commercial. See: http://creativecommons. org/licenses/by-nc/4.0. 